\title{
Direct detection and imaging of low-energy electrons with delta-doped charge-coupled devices
}

\author{
Shouleh Nikzad, Qiuming Yu, ${ }^{\text {a) }}$ Aimée L. Smith, ${ }^{\text {b) }}$ Todd J. Jones, T. A. Tombrello, ${ }^{c)}$ \\ and S. Tom Elliott \\ Center for Space Microelectronic Technology, Jet Propulsion Laboratory, California Institute of \\ Technology, Pasadena, California 91109
}

(Received 24 July 1998; accepted for publication 6 October 1998)

\begin{abstract}
We report the use of delta-doped charge-coupled devices (CCDs) for direct detection of electrons in the 50-1500 eV energy range. We show that modification of the CCD back surface by molecular beam epitaxy can greatly improve sensitivity to low-energy electrons by introducing an atomically abrupt dopant profile to eliminate the dead layer. Using delta-doped CCDs, we have extended the energy threshold for detection of electrons by over an order of magnitude. We have also measured high gain in response to low-energy electrons using delta-doped CCDs. The effect of multiple electron hole pair production on the observed signals is discussed. Electrons have been directly imaged with a delta-doped CCD in the $250-750 \mathrm{eV}$ range. (c) 1998 American Institute of Physics.
\end{abstract}

[S0003-6951(98)03549-9]

There is great interest in detecting and imaging electrons, especially low-energy electrons (tens of eV to thousands of eV) for scientific spectroscopy applications, such as low-energy electron diffraction spectroscopy and reflection electron energy-loss spectroscopy at reflection high-energy electron diffraction energies. ${ }^{1,2}$ In addition, there are space science applications for low-mass, low-power plasma detectors and imagers. Imaging systems for low-energy particles generally use microchannel plate electron multipliers followed by position-sensitive solid-state detectors, or phosphors and position-sensitive photon detectors. These systems work well and can process up to $10^{6}$ electrons/s; however, they have difficulties with gain stability, require high voltages, and the dynamic range and spatial resolution of these compound systems is considerably less than that of a solidstate imaging detector.

Because of their high resolution, linearity, and large dynamic range, silicon charge-coupled devices (CCDs) could make major advances in particle detection. CCDs have been used to meet the needs of a wide range of scientific imaging applications which require accurate photometric imaging at low light levels with high dynamic range. They have been remarkably successful as imagers of x-ray, UV, visible, and near-IR photons. ${ }^{3}$ As low-energy particle detectors and imagers, CCDs can make a great impact in many scientific fields. However, their use as particle detectors has been hampered by the inherent problems existing in the frontsideilluminated CCDs. Both the rapid radiation degradation caused by energetic electrons passing through the frontside gates and gate insulator structure, and the large dead layer to the low-energy electrons presented by the thick frontsidegate structure make frontside-illuminated CCDs unsuitable as electron detectors.

While backside-illuminated, thinned CCDs offer the

\footnotetext{
${ }^{a)}$ Currently at Kansas State University, Manhattan, KS 66506.

${ }^{b)}$ Currently at Massachusetts Institute of Technology, Cambridge, MA 02139.

c) Also with the Division of Physics, Mathematics, and Astronomy, California Institute of Technology, Pasadena, CA 91125.
}

possibility of detecting low-energy electrons, they inherently possess a back surface dead layer associated with the backside potential well (caused by positive charge at the interface between $\mathrm{Si}$ and $\mathrm{SiO}_{2}$ ). The problem is similar to the detection of UV photons because a significant fraction of the energy of incident electrons is deposited within a few hundred $\mathrm{nm}$ of the surface (e.g., $100 \%$ of energy lost within $17 \mathrm{~nm}$ for $1 \mathrm{keV}$ electron beam ${ }^{4}$ ). A number of techniques have been explored to eliminate the backside potential well, such as negative-surface charging or biasing (e.g., UV flooding and bias flash gating) and ion implantation. ${ }^{5,6}$ In previous studies, untreated and treated backside-thinned CCDs have been used for electron detection, with promising results for electrons in the $1-20 \mathrm{keV}$ range.$^{5-8}$ For example, the results of the biased flash-gate CCD study show that the average quantum efficiency increases from less than $1 \%$ for an untreated CCD to nearly $40 \%$ for a backside-treated CCD at an electron-beam energy of $1 \mathrm{keV}$. Although these backside surface treatments have generated good electron or UV quantum efficiency, they suffer variously from problems of yield, response stability, hysteresis, and long-term reliability. All of these problems critically affect the detection of low-energy electrons.

Delta-doped CCDs have the potential to detect electrons at significantly lower energies than previously possible because the dead layer associated with the backside potential well has been eliminated. ${ }^{9}$ Delta-doped CCDs with $100 \%$ internal quantum efficiency in the visible and UV were developed at the Jet Propulsion Laboratory. ${ }^{9}$ Further studies have shown that the delta-doped CCDs are highly uniform and that these devices exhibit long-term stability. ${ }^{10}$ In this approach, only a few atomic layers of silicon, containing an extremely high concentration of $p$-type dopant (at least 2 $\times 10^{14}$ boron atoms $/ \mathrm{cm}^{2}$ ) are epitaxially grown on the CCD backside surface, using molecular beam epitaxy (MBE). The spiked concentration of dopant atoms permanently pins the conduction band at the back surface, eliminating the dead layer and creating a built-in field driving free electrons to the collection wells under the frontside gates.

Fully processed EG\&G Reticon CCDs (512 pixels $\times 512$ 
pixels, $27 \mu \mathrm{m}$ pixel) were modified by low-temperature molecular beam epitaxy following the process described in previous papers. ${ }^{9,11}$ To make direct comparisons between an untreated $\mathrm{CCD}$ and a delta-doped CCD, a CCD that was partially masked during the MBE process was used in our measurements.

We report direct imaging of low-energy electrons using CCDs. Electrons in the $250-750 \mathrm{eV}$ range were imaged with CCDs in a custom ultrahigh vacuum (UHV) chamber. A camera was mounted directly onto the UHV chamber to operate the CCD in the imaging mode. A modified SONY cathode ray tube (CRT) with an indirectly heated cathode gun and beam energy range of 200-2000 eV served as the source of incident electrons with negligible light background. The imaging mode of operation allows for observation of electron irradiation on operating parameters such as chargetransfer efficiency (CTE), individual pixel response, and surface charging. Because of the highly sensitive imaging mode of operation, the incoming flux of electrons was controlled with a mechanical shutter, with beam exposure times in the range $0.01-2 \mathrm{~s}$.

The difference in the response between a delta-doped CCD and an untreated CCD is most apparent in the response of the CCD that was partially masked during the MBE process as shown in Fig. 1. Flat-field images of $500 \mathrm{eV}$ electrons with the delta-doped CCD shows excellent qualitative similarity to UV images at $250 \mathrm{~nm}$, with nearly identical contrast between the delta-doped and control regions of the CCD. The absorption length of $250 \mathrm{~nm}$ light in silicon is approximately $70 \AA,^{12}$ and electrons with $500 \mathrm{eV}$ energy have a maximum penetration depth of $50 \AA \AA^{4,13}$ Some small dark blemishes are apparent in the electron flat-field image that are not seen on the UV flat field, most likely due to dust or debris that has been introduced to the membrane surface in the course of handling, transporting, and storing the device in the months following the date when the UV flat-field image was taken. Additional studies of electron imaging with the delta-doped CCDs are under way.

Quantum efficiency measurements for electrons in the $50-1000 \mathrm{eV}$ energy range were performed with the CCD configured as a photodiode and using three different sources of electron beam: an indirectly-heated cathode gun described above; a directly-heated cathode gun, (both mounted in the custom UHV system); and the electron source in a scanning electron microscope (SEM). In photodiode mode, a CCD is operated in such a way as to integrate the entire signal collected over the surface of the device by grounding all pins except for the output amplifiers. The signal is then read from the pin of one of the output amplifiers, giving the total response of all the pixels in the irradiated region of the device indicating the overall collection efficiency. By integrating the response of all irradiated pixels, much of the error that would result in a pixel-by-pixel measurement is effectively averaged out. The ratio of CCD output current divided by incident beam current (measured by a Faraday cup) was used to measure the quantum efficiency of the device.

In the custom ultrahigh vacuum chamber, a Faraday cup and phosphor screen were mounted vertically on a manipulator from top to bottom, facing the electron beam. Both the Faraday cup and CCD outputs were measured with a digital Downloaded 16 Dec 2005 to 131.215.225.9. Redistribution subject
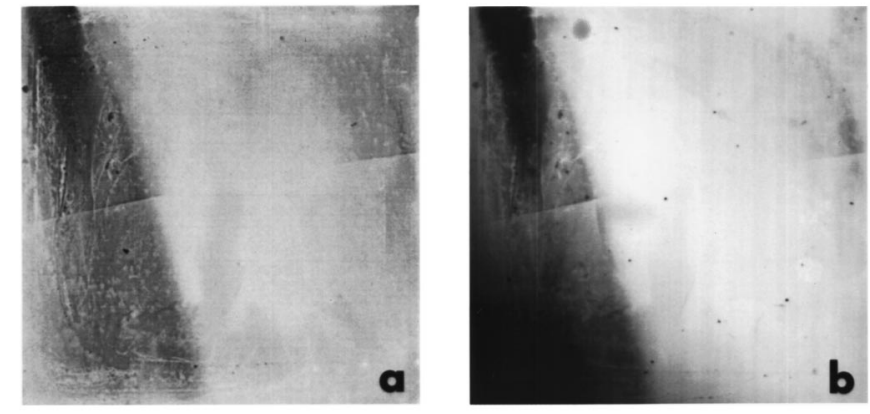

FIG. 1. Images of a partially masked delta-doped CCD with $250 \mathrm{~nm}$ uniform light (a) and $500 \mathrm{eV}$ electrons (b). Brightness in the two images is adjusted differently; however, qualitatively the two images are the same. The dark area on the left of both images is untreated (masked during MBE growth) and the delta-doped region is bright, indicating sensitivity to UV photons and electrons. The electron beam was slightly off center and a portion of the CCD corresponding to the bottom-left corner of the image was not exposed to the electron beam.

voltmeter through an amplifier with $10.5 \mathrm{mV} / \mathrm{nA}$ gain. During the measurements with the indirectly-heated cathode gun the electron beam was first focused into a $\sim 1 \mathrm{~mm}$ spot in the center of the phosphor screen, then the Faraday cup and the $\mathrm{CCD}$ were moved down in sequence to measure the current of the incident electron beam and the CCD output current, respectively. After the CCD measurement, the incident electron-beam current was measured again using the Faraday cup to check the beam stability. To ensure that both the CCD and the Faraday cup were exposed to the same area of electron beam, an aperture with diameter of $0.64 \mathrm{~cm}$ was centered in front of each. A plate with negative bias was put in front of the Faraday cup to repel secondary electrons. Typical beam currents were in the hundreds of $\mathrm{pA}$ range. The typical vacuum levels during the measurements were in the low $7 \times 10^{-8}-2 \times 10^{-7}$ Torr range. A directly-heated cathode gun or a flood gun was used as a source of electrons with energies in the tens of $\mathrm{eV}$. This electron source also produces a large light background that was distinguished for the electron signal by measuring the CCD response before and after magnetically deflecting the electron beam. For this reason, the response of the delta-doped CCD in this energy range is only reported qualitatively. Another set of measurements were performed in a SEM to take advantage of its highly focused electron beam for mapping the response of the CCD. The SEM measurements were performed using a JEOL JSM6400 SEM as the source of the electrons, which could provide beam energies from 0.2 to $40 \mathrm{keV}$. Both the beam current and the CCD output current were measured with a Keithley 485 picoammeter. The incident beam current was measured with a Faraday cup that could be rotated in and out of the beam path in front of the CCD. The beam current for the measurements were in the range of $2-40 \mathrm{pA}$. The pressure during the measurements was approximately $10^{-5}$ Torr.

The responses of a delta-doped CCD and an untreated backside-thinned CCD to electrons were repeatedly measured in the range of $200-1000 \mathrm{eV}$ using the modified CRT and the SEM as sources. In Fig. 2, the CCD quantum efficiency is plotted as a function of electron incident energy. Quantum efficiency was calculated by dividing the measured current from the CCD configured in photodiode mode to the measured electron-beam current (measured by a Faraday to AIP license or copyright, see http://apl.aip.org/apl/copyright.jsp 


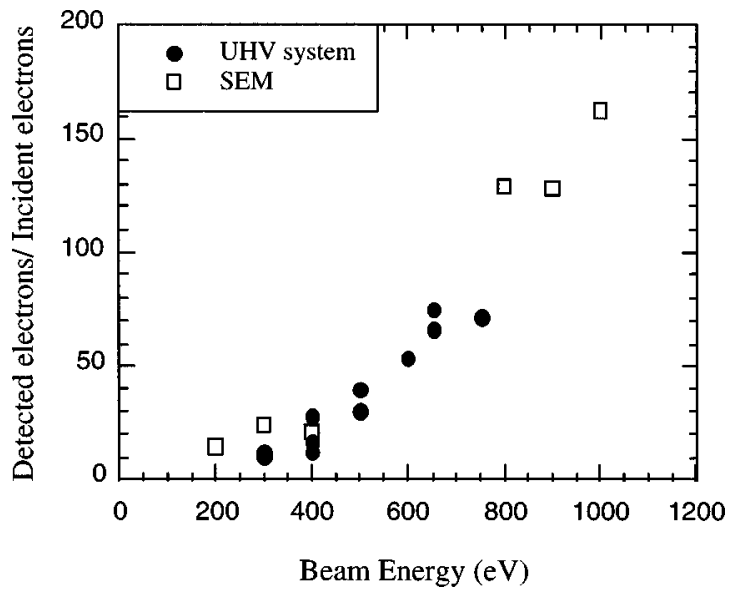

FIG. 2. Ratio of detected electrons to incident electrons as a function of energy. The response of the CCD increases with increasing energy as a result of multiple electron-hole pair generation.

cup), which is equivalent to the number of electrons detected divided by the number of incident electrons. Because portions of the delta-doped CCD were masked during processing to serve as control regions, data taken in the UHV system were corrected to account for the fraction of untreated exposed CCD area. The untreated backside-thinned CCD showed a dramatically lower quantum efficiency than the delta-doped CCD. The response of the untreated CCD to electrons was unstable, decaying with a time constant on the order of $20 \mathrm{~min}$ at an incident electron energy of $1 \mathrm{keV}$ and it showed no response at $300 \mathrm{eV}$. The measured quantum efficiency of the delta-doped CCD increases with increasing energy of the incident beam. The dependence of quantum efficiency on incident energy is due to the complicated interaction of electrons with silicon, which results in the generation of multiple electron-hole pairs in the cascade initiated by each incident electron. Multiple electron-hole pair production, also known in the literature as quantum yield, is observed in the measured UV and x-ray response of deltadoped CCDs and other devices. Quantum yield greater than unity has been previously observed in backside-illuminated CCDs modified using both the flash-gate ${ }^{5}$ and ion implantation ${ }^{14}$ techniques at electron energies greater than 1 $\mathrm{keV}$.

To the best of our knowledge, the delta-doped CCD is the first CCD shown to respond to electrons with energies lower than $300 \mathrm{eV}$. At $1 \mathrm{keV}$, which is the previously reported lower limit for the flash-gate $\mathrm{CCD},{ }^{5}$ the quantum efficiency of the delta-doped CCD is approximately twice as great. The delta-doped CCD exhibited a response above the noise at electron energies as low as $50 \mathrm{eV}$. The minimum energy tested at this point represents source limitations rather than detector limitations.

Analogous to photon quantum efficiency of CCDs, electron quantum efficiency (QE) is the product of three quantities: the transmission coefficient, the quantum yield, and the internal quantum efficiency. ${ }^{15}$ The transmission coefficient is a factor representing the fraction of incident beam absorbed in the device, which for electrons includes the backscattering coefficient; the quantum yield accounts for the statistically averaged number of electron-hole pairs produced by the incident electron (or photon); and the internal quantum effi- ciency accounts for internal losses in the CCD, such as recombination of electron-hole pairs at the back surface of the CCD. ${ }^{14}$ Ultraviolet measurements of the delta-doped CCD indicate that the internal quantum efficiency is nearly $100 \%$, even at $270 \mathrm{~nm}$ where the absorption length in silicon is only $4 \mathrm{~nm} .{ }^{9}$ Assuming that all the generated electrons are detected by the delta-doped CCD (internal QE $\sim 100 \%$ ), our measurements will represent the product of the effective quantum yield $^{16,17}$ of silicon and the transmission factor for lowenergy electrons. If the transmission factor is dominated by the backscattering coefficient, i.e., $40 \%-50 \%{ }^{18}$ for $200-$ $1500 \mathrm{eV}$ electrons, the effective quantum yield can be determined from our measurements.

While separating the effects of transmission and quantum yield is interesting from a theoretical standpoint, the convolution of the two, as measured in these experiments, is the quantity of interest for solid-state electron detectors. It is significant that, to the best of our knowledge, no other solidstate devices detect low-energy electrons as efficiently as the delta-doped CCD, due to the presence of a dead layer near their surfaces. In addition to its high efficiency, the deltadoped CCD also has the capability of imaging low-energy particles, which may prove valuable in energy-selective particle detector applications.

The authors gratefully acknowledge the invaluable assistance of Dr. M. E. Hoenck, Dr. L. D. Bell, Dr. S. Manion, Dr. T. Van Zandt, W. Proniawicz, and Professor L. C. Kimerling. The work presented in this letter was performed by the Center for Space Microelectronics Technology, Jet Propulsion Laboratory, California Institute of Technology, and was jointly funded by the Caltech President's Fund and the NASA Office of Space Science.

${ }^{1}$ S. Nikzad, S. S. Wong, C. C. Ahn, A. L. Smith, and H. A. Atwater, Appl. Phys. Lett. 63, 1414 (1993).

${ }^{2}$ S. Nikzad, C. C. Ahn, and H. A. Atwater, J. Vac. Sci. Technol. B 10, 762 (1992).

${ }^{3}$ J. R. Janesick, S. T. Elliott, S. A. Collins, H. Marsh, M. M. Blouke, and J. Freeman, Opt. Eng. (Bellingham) 26, 692 (1987).

${ }^{4}$ T. E. Everhart and P. H. Hoff, J. Appl. Phys. 42, 5837 (1971).

${ }^{5}$ T. Daud, J. R. Janesick, K. Evans, and T. Elliott, Opt. Eng. (Bellingham) 26, 686 (1987).

${ }^{6}$ M. K. Ravel and A. L. Reinheimer, Proc. SPIE 1447, 109 (1991).

${ }^{7}$ J. C. Richard and M. Vittot, Nucl. Instrum. Methods Phys. Res. A 315, 368 (1992).

${ }^{8}$ K. L. Luke and L.-J. Cheng, J. Appl. Phys. 60, 589 (1986).

${ }^{9}$ M. E. Hoenk, P. J. Grunthaner, F. J. Grunthaner, M. Fattahi, H.-F. Tseng, and R. W. Terhune, Appl. Phys. Lett. 61, 1084 (1992).

${ }^{10}$ S. Nikzad, M. E. Hoenk, P. J. Grunthaner, R. W. Terhune, F. J. Grunthaner, R. Wizenread, M. Fattahi, and H.-F. Tseng, Proc. SPIE 2198, 907 (1994).

${ }^{11}$ M. E. Hoenk, P. J. Grunthaner, F. J. Grunthaner, R. W. Terhune, and M. Fatthi, Proc. SPIE 1656, 488 (1992).

${ }^{12}$ S. M. Sze, Physics of Semiconductor Devices, 2nd ed. (Wiley, New York, 1981), p. 42.

${ }^{13}$ S. Nikzad, A. L. Smith, S. T. Elliott, T. J. Jones, T. A. Tombrello, and Q. Yu, Proc. SPIE 3019, 241 (1997).

${ }^{14}$ D. G. Stearns and J. D. Wiedwald, Rev. Sci. Instrum. 60, 1095 (1989).

${ }^{15}$ S. Nikzad, M. E. Hoenk, P. J. Grunthanber, R. W. Terhune, R. Wizenread, M. Fattahi, H.-F. Tseng, and F. J. Grunthaner, Proc. SPIE 2217, 355 (1994)

${ }^{16}$ C. A. Klein, J. Appl. Phys. 39, 2029 (1968).

${ }^{17}$ L. R. Canfield, J. Kerner, and R. Korde, Appl. Opt. 28, 3940 (1989).

${ }^{18}$ A. L. Smith, Q. Yu, S. T. Elliott, T. A. Tombrello, and S. Nikzad, Mater. Res. Soc. Symp. Proc. 448, 177 (1996). 